\title{
Remission of Hypnic Headache Associated with Idiopathic Cyclic Edema with the Use of Aminaphtone
}

\author{
José Maria Pereira de Godoy*
}

\author{
Department Cardiology and Cardiovascular Surgery in Medicine School São José do Rio Preto-FAMERP and CNPq \\ (National Council for Research and Development), Brazil
}

\begin{abstract}
Headache is one of the chief complaints among patients visiting primary care physicians. The aim of the current study is to report on a case of daily intermittent headaches in a patient with idiopathic cyclic edema. Over three years the patient suffered from headaches that started and improved at specific times. They began at about 5 to 6 o'clock in the morning and spontaneously disappeared 3 to 4 hours after getting up and thus were directly associated to sleep. The patient complained of generalized edema and had weight differences of about two kilograms between 7 a.m. and 5 p.m. A hypothesis of idiopathic cyclic edema was reached and the patient was prescribed aminaphtone which cured the headaches within two days after initiating treatment.
\end{abstract}

Keywords: Intermittent headache, aminaphtone, treatment.

\section{INTRODUCTION}

Headache is one of the chief complaints among patients visiting primary care physicians. Diagnosis begins with the exclusion of secondary causes for headache. More than $90 \%$ of patients have a primary-type headache, so diagnosis can often be completed without further testing [1]. Migraine is a common chronic, intermittent headache disorder that in some patients is accompanied by neurological symptoms, particularly visual symptoms, known as migraine aura [2]. Many women experience headaches, including migraine, in association with their menstrual cycles. Although definitions vary, menstrual migraine generally refers to migraine without aura that occurs within several days prior to and several days after the onset of menses [3]. The most common menstrual disorders are dysmenorrhea and headache; these conditions are the leading causes of work or school absenteeism and substantially impact quality of life. Headache associated with menses is often migraine and referred to as menstrual migraine [4]. Although the different conditions of headaches are well defined, there are conditions that are not emphasized such as headache associated with idiopathic cyclic edema.

Idiopathic cyclic edema syndrome was identified in 1955 by Mach and relates to a set of clinical states that involve vascular hyperpermeability associated to edema due to interstitial retention of fluid [5]. Despite all the changes, this disease is self-limiting with the variations in weight during the day being very apparent with edema of the legs and ankles and sometimes of the eyelids, face and abdomen [6,7]. The aim of the current study is to report a case of intermittent daily headache of a patient with idiopathic cyclic edema treated using aminaphtone.

*Address correspondence to this author at the Rua Floriano Peixoto, 2950, São José do Rio Preto, CEP: 15010-020, SP, Brazil; Tel: 551732326362; E-mail: godoyjmp@riopreto.com.br

\section{CASE REPORT}

A 56-year-old patient reported a history of generalized edema and headache over three years. She complained that each day she woke up in the morning with the face and hands swollen which gradually improved after getting up however edema of the legs developed by the evening. The edema of the face was visible and of the hands was characterized by difficulty to bend the fingers and to take off rings in the morning with the necessity of removing them before sleeping. Associated with the edema the patient reported daily headaches that started at about 5:00 a.m. and improved around 3 to 4 hours after getting up and thus had a clear association with sleep. When the patient did not lie down at night to sleep, she did not suffer from headaches. However, when she did, shortly before awakening she suffered from headaches. The pain is described as a dull pain with the patient becoming irritated and with increased sensitivity. The patient denies nausea, vomiting, visual symptoms, autonomics symptoms such as redness or tearing of the ears or nares congestion or drainage, but has sensitivity light and noise.

In this period, the patient was submitted to a hysterectomy. The patient consulted in several medical centers across the country but without finding any solution for the headaches. She performed several examinations but none identified alterations.

Soft painless edema of the lower limbs was observed in the physical examination. The patient was requested to weigh herself at 7:00 a.m. and 5:00 p.m. for three days and to return to the clinic afterwards taking into account the amount of food ingested in this period. The patient reported a weight difference of $2 \mathrm{~kg}$ between the morning and evening. A hypothesis of idiopathic cyclic edema was reached and $75 \mathrm{mg}$ aminaphtone was prescribed three times daily.

The patient reported that she needed to get up three times during the first night for diuresis. In two days the headache, generalized edema and the symptoms disappeared. Two 
years later, the patient continues to take two tablets of aminaphtone per day. Despite of guidance from the physician to suspend the drug the patient is concerned about the possibility of the symptoms returning.

\section{DISCUSSION}

The current study reports on a novel clinical treatment for headaches resulting from idiopathic cyclic edema, a situation rarely mentioned in publications. An intermittent character of the headache was observed; the patient identified the time the headache appeared as 5:00 a.m. and its disappearance a few hours after she got up, even without taking medications. She described it as a pain that had a time to appear and disappear which was associated with sleep. However, what drew our attention was the difficulty to establish a diagnosis and an efficacious treatment.

The clue to the diagnosis of idiopathic cyclic edema is the description of generalized edema; as many patients do not notice these signs it is important to consider this possibility [5]. Capillary permeability measured using the Landis isotope test may be performed. The history of a change in weight of more than $800 \mathrm{gm}$ between the morning and the evening (12 hours of evaluation), edema of the face on waking up and the difficulty to remove rings are all suggestive of idiopathic cyclic edema but other types of edema should also be considered [5].

The physiopathology of this type of headache is a change in the capillary permeability which also leads to generalized edema with displacement of edema due to gravitational pressure. During the day the edema concentrates in the lower extremities and during sleep, redistribution of edema occurs, resulting in swelling of the upper limbs, head and brain. Thus the origin of the headache is brain edema.

In the literature one type of headache, hypnic headache, is associated with sleep however its physiopathology is not well understood [8]. In the current study the association of headaches with cyclic edema and with the control of edema was well characterized. Thus, we suggest that the possibility of the involvement of cyclic edema in hypnic headaches should be investigated which may throw light on this type of headache.

The treatment and control of idiopathic cyclic edema is attained by drugs such as aminaphtone that interfere in the capillary permeability. However there is no specific therapy as the cause of idiopathic cyclic edema remains unknown. Aminaphtone controls the disease well in about $70 \%$ of cases, gives an improvement in around $20 \%$ of patients and is inefficacious in $10 \%$ [5]. In cases where control is unsatisfactory the option is to try other drugs, either in association or isolated. One of the options is ginkgo biloba which, at doses of 80 to $120 \mathrm{mg}$, can provide an improvement in $90 \%$ of cases. A aminaftone é droga utilizada

Another aspect in these patients is the association of symptoms due to edema such as pain in the legs and body which generally improve with control of the edema. This patient illustrates the difficulties in the treatment and the improvement of the other symptoms.

\section{REFERENCES}

[1] Mueller LL. Diagnosing and managing migraine headache. J Am Osteopath Assoc 2007; 107(10 Suppl 6): ES10-6.

[2] Kurth T. Associations between migraine and cardiovascular disease. Expert Rev Neurother 2007; 7(9): 1097-104.

[3] Mannix LK, Files JA. The use of triptans in the management of menstrual migraine. CNS Drugs 2005; 19(11): 951-72.

[4] Mannix LK. Menstrual-related pain conditions: dysmenorrhea and migraine. J Womens Health (Larchmt) 2008; 17(5): 879-91.

[5] Pereira de Godoy JM. Aminaphtone in idiopathic cyclic oedema syndrome. Phlebology 2008; 23(3): 118-9.

[6] Yucha C, McKay S. Idiopathic edema. ANNA J 1992; 19(1): 2932.

[7] Anand AC, Narula AS, Anand M, Mishra A, Singh. Idiopathic oedema - a missed entity. J Assoc Phys India 1991; 39(3): 258-9.

[8] Lanteri-Minet M, Donnet A. Hypnic Headache. Curr Pain Headache Rep 2010. [Epub ahead of print].

Received: March 06, 2010

Revised: May 31, 2010

Accepted: June 21, 2010

(C) José Maria Pereira de Godoy; Licensee Bentham Open.

This is an open access article licensed under the terms of the Creative Commons Attribution Non-Commercial License (http://creativecommons.org/licenses/by-nc/3.0/) which permits unrestricted, non-commercial use, distribution and reproduction in any medium, provided the work is properly cited. 